\title{
Geschichtsbewusstsein und Zeitzeugnis. Nietzsches Genealogie als Anregung zum philosophischen Dialog mit Kindern
}

\author{
Eva Marsal (Pädagogische Hochschule, Karlsruhe) \\ Takara Dobashi (Hiroshima Universität, Hiroshima)
}

\section{Das Leben als Geltungskriterium der Historie}

\begin{abstract}
»Uebrigens ist mir Alles verhaßt, was mich bloss belehrt, ohne meine Thätigkeit zu vermehren oder unmittelbar zu beleben«. Das sind Worte Goethes, mit denen. als mit einem herzhaft ausgedrückten Ceterum censeo, unsere Betrachtung über den Werth und den Unwerth der Historie beginnen mag. In derselben soll nämlich dargestellt werden, warum Belehrung ohne Belebung, warum Wissen, bei dem die Tätigheit erschlafft, warum Historie als kostbarer Erkenntnis-Ueberfluss und Luxus und ernstlich, nach Goethes Wort, verhasst sein muß - deshalb, weil es uns noch am Nothwendigsten fehlt, und weil das Ueberflüssige der Feind des Nothwendigen ist. Gewiß, wir brauchen Historie, aber wir brauchen sie anders, als sie der verwöhnte Müssiggänger im garten des Wissens braucht, mag derselbe auch vornehm auf unsere derben und anmuthlosen Bedürfnisse und Nöthe herabsehen. Das heisst, wir brauchen sie zum Leben und zur That, nicht zur bequemen Abkehr vom Leben und von der That, oder gar zur Bechönigung des selbstsüchtigen Lebens und der feigen und schlechten That. Nur soweit die Historie dem Leben dient, wollen wir sie dienen: aber es giebt einen Grad, Historie zu treiben, und eine Schätzung derselben, bei der das Leben verkümmert und entartet (Nietzsche, KSA I, 245).
\end{abstract}

Dieses Zitat des jungen Nietzsche findet sich in der kleinen Schrift Vom Nutzen und Nachtheil der Historie für das Leben, die er im Rahmen seiner Unzeitgemäßen Betrachtungen herausgab. Hier stellte er die aufstrebende Disziplin "Geschichtswissenschaft" auf den Prüfstand, die als eine objektive Größe für Nietzsches Zeitgenossen sakrosankt ist: 
Unzeitgemäss ist auch diese Betrachtung, weil ich etwas, worauf die Zeit mit Recht stolz ist, ihre historische Bildung hier einmal als Schaden, Gebreste und Mangel der Zeit zu verstehen versuche (ibid. 246).

Die wissenschaftliche Erkenntnis scheint im 19. Jahrhundert nach ihrer Emanzipation von der Theologie über jeden Zweifel erhaben zu sein und durch die Heraufkunft der wissenschaftlich-technischen Welt mit ihren ungeheuren Erleichterungen als Garant für das Glück der Menschheit zu gelten. „Fortschritt“ heißt der neue Hoffnungsbegriff, der die sokratischplatonische Entsprechung von dem „Wahren und Guten“ zu einem technisch einlösbaren Versprechen werden ließ. „Fortschrittserwartung und Wissenschaftsglaube verschmolzen zum wirkungsmächtigen politischen Motiv nicht nur im liberalen Bürgertum, sondern auch in der Arbeiterbewegung" (Gerhardt 1995, 96). Die als sakrosankt geltende Objektivierung macht auch vor der aufstrebenden Geschichtswissenschaft nicht halt, die nun im Sinne des Menschheitsfortschritts als Verheißung der kontinuierlichen Steigerung von Wohlstand und Glück verstanden wird. Die politischen Katastrophen des 20. Jahrhunderts sowie die durch die Globalisierung verursachten sozialen, ökonomischen und ökologischen Krisen waren zu dieser Zeit nicht vorstellbar. In seiner Schrift über den Sinn bzw. Unsinn der Historie für das Leben entlarvt Nietzsche die ideologische Fortschrittseuphorie des 19. Jahrhunderts als Selbsttäuschung und oberflächliche Selbstgefälligkeit einer jungen wissenschaftlichen Zivilisation, die sich selbst als „Ziel und befriedigendes Ende der Geschichte präsentiert“ (ibid. 96). Nietzsche ist sich der Unaufhaltsamkeit aller Veränderungen bewusst und damit der Notwendigkeit, sich um Fortschritt zu bemühen, damit die zivilisatorischen, d.h. vor allem selbstgeschaffenen Probleme gelöst werden, deren Lösungen aber unausweichlich neue Risiken in sich bergen. Im Gegensatz zu seiner Zeit ist sich Nietzsche der Tragik des Einzelnen und der Menschheit klar, die sich daraus ergibt, dass der Mensch nicht nur Geschichte hat, sondern auch in jedem Augenblick Geschichte macht und damit seine Kultur und seine Zeit. Der Mensch hat durch seine Fähigkeit „Sich-zuErinnern“ und "Sich-in-Distanz-zu-sich“ zu setzen einen Zugang zu seiner Geschichte und der kommende Zeit. Erst mit der bewussten Tat, die mit dem Gedächtnis verbunden ist, entsteht das Kontinuum von Vergangenheit, Gegenwart und Zukunft.

\subsection{Der subjektive Perspektivismus des Denkens}

Da „Bewusstsein“ ein relationaler Begriff ist und deshalb mit einem kollektiven oder individuellem Subjekt verbunden sein muss, ist jeder Geschichtsbezug, der nach Nietzsche einen Wert besitzt, mit einer einzigartigen Bedeutung für den sich darauf Beziehenden verbunden und wird 
aus einer einzigartigen Perspektive heraus verstanden. Deshalb erteilt Nietzsche der zu seiner Zeit aufkommenden Objektivierung der historischen Wissenschaft eine Absage. Gegen den objektivierten „Erkenntnis-Überfluss“ setzt Nietzsche als Ausdruck seiner experimentalen Philosophie der Gegensätze das Spiel mit den Widersprüchen, das sich wissenschaftstheoretisch als ein Methodenpluralismus interpretieren lässt und von Nietzsche durch den "Perspektivismus“ des Daseins gerechtfertigt wird. „Das ,Perspektivische' ist für Nietzsche die ,Grundbedingung des Lebens' (wie es in der Einleitung zu Jenseits von Gut und Böse heisst), da jedes Lebewesen lediglich seinen spezifischen Ausschnitt von der Realität erfassen kann, diesen aber gleichwohl für das Ganze hält. Insofern ist der Perspektivismus ,nur eine komplexe Form der Spezifität', also die Form der Besonderheit, die jedem Wesen unvermeidlich zukommt (KSA 9, 14/186; 13, 372). Da aber die eigenen Perspektiven die Parameter der Wahrnehmung bilden, lassen sie sich kaum selbst erfassen und übersteigen. Auch folgt aus Nietzsches Gedanken, dass es keine allumfassende Gesamtperspektive gibt, an der alle partizipieren oder die alle Perspektiven umspannt. Die jeweilige Perspektive bestimmt die Interpretation aller Begriffe. Selbst die sozial und kulturell geteilten Begriffe wie Welt oder Wirklichkeit sind davon nicht ausgenommen, sondern lassen sich lediglich als Ausdruck einer perspektivischen Einstellung verstehen, in der eine Person alle Erkenntnisse zusammenfasst, die sie innerhalb ihrer Position begreifen kann. „Folglich ist alles Erkennen an Perspektiven gebunden; der Mensch kommt über den zu seiner leiblichen Organisation gehörenden intellektuellen Horizont nicht hinaus" (Gerhardt 1995, 138). In seiner Aphorismensammlung Die Fröhliche Wissenschaft fasst Nietzsche zusammen: „Wir können nicht um unsre Ecke sehn: es ist eine hoffnungslose Neugierde wissen zu wollen, was es noch für andre Arten Intellekt und Perspektive geben könnte" (KSA 4, 374; 3, 626). Mit dieser Theorie greift er einen Gedanken seines sprachphilosophischen Großvaters auf. So vermerkte der Superintendent und Nietzsches Grossvater, Friedrich August Ludwig Nietzsche, in seinem 1796 erschienen Werk Gamaliel, oder über die immerwährende Dauer des Christenthums:

Nie hat es, so lange die Welt steht, noch zwischen Menschen gegeben, die sich ein und derselben Sache, sie mochte betreffen was sie wollte, einerley Vorstellungen und begriffe gebildet hätten. Dieser stellte sich die Sache von dieser Seite, und jener wieder von einer andern vor.

Während dieses Verständnis aber an den perspektivistischen Objektivismus von Leibniz anknüpft, der von einer objektiven Wirklichkeit ausgeht, die aufgrund der unterschiedlichen Standpunkte und Eigenschaften der unterschiedlichen Betrachter in unterschiedlichen Ansichten gegeben ist, 
entwirft sein Enkelsohn den perspektivistischen Subjektivismus, der die Pluralität der Wirklichkeiten voraussetzt (KSA 5, 365).

\subsection{Nietzsches Bildungstheorie}

Nietzsche kritisiert an der zu seiner Zeit hochgeschätzten Geschichtswissenschaft, dass sie durch die Forderung „Wissenschaft zu sein“ die "natürlich fliesende Beziehung zwischen Leben und Historie“, aufgekündigt hat (KSA I, 271). Dadurch wird bei der Geschichtsrezeption die „Perspektivität“ von Bildung ausgeblendet und jeder dazu gezwungen, standardisiertes Wissen aufzunehmen, dass sich in ihm anstaut, da es in keiner Beziehung zu ihm als dem Empfangenen steht und durch seine zusammenhangslose Anhäufung nicht belebt, sondern belastet. Einen Unwert bis hin zum Schaden besitzt die Historie als Wissenschaft nach Nietzsche also dann, wenn ihr Zweck nicht mehr darin besteht, als Ressource für das Leben $\mathrm{zu}$ dienen, sondern wenn sie ich fern als Wissensstoff, als Faktenwissen, angeeignet werden muss. Die Nutzlosigkeit dieses Vorgangs pointiert Nietzsche mit der Grimmschen Wackersteinmetapher „Der moderne Mensch schleppt zuletzt eine ungeheure Menge von unverdaulichen Wissenssteinen mit sich herum, die dann im Leib rumpeln, wie es im Märchen heisst“" (KSA I, 272).

Gegen diese Bildungstheorie ruft Nietzsche der Jugend zu: „Der Mensch, welcher nicht zur Masse gehören will, braucht nur aufzuhören, gegen sich bequem zu sein; er folge seinem Gewissen, welches ihm zuruft: 'sei du selbst! Das bist du alles nicht, was du jetzt thust, meinst, begehrst'“ (ibid. 338).

Nietzsche, der sich als Erzieher der Deutschen verstand, sah seine Aufgabe darin, die Jugendlichen zum Selbstdenken im Sinne der Vervollkommnung und Übersteigung der eigenen Lebensmöglichkeiten und zur Verfolgung hochgesteckter Zielen zu ermutigten. In Rückgriff auf Goethes „edelsten Bildungskampfe“ (KSA I, 129-131) fordert Nietzsche eine Schule, die jungen Leuten ermöglicht selbstständige Urteilskraft herauszubilden und ihre wegweisende Vernunft zu fördern. Deshalb propagiert er:

Die Vernunft in der Schule. - Die Schule hat keine wichtigere Aufgabe, als strenges Denken, vorsichtiges Urtheilen, consequentes Schliessen zu lernen: deshalb hat sie von allen Dingen abzusehen, die nicht für diese Operationen tauglich sind, zum Beispiel von der Religion. Sie kann ja darauf rechnen, dass menschliche Urtheilkraft, Gewöhnung und Bedürfnis später doch wieder den Bogen des allzu straffen Denkens abspannen. Aber so lange ihr Einfluss reicht, soll sie Das erzwingen, was das Wesentliche und Auszeichnende am Menschen ist: "Vernunft und Wissenschaft des Menschen allerhöchste Kraft" Wie wenigstens Goethe urtheilt (KSA 2, 220). 
Dieses Zitat zeigt deutlich, dass Nietzsche keinesfalls wissenschaftsfeindlich eingestellt ist, sondern sich lediglich gegen ihre blinde Verehrung wendet, die den kritischen Blick auf die möglichen Sekundärschäden außer acht lässt. Sein Anspruch liegt in der Lebensdienlichkeit aller Wissenschaft, vor allem aber der historischen, denn diese ist stets im Menschsein enthalten, Handeln ist seinem Wesen nach geschichtlich. „Der Bezug zur Vergangenheit gehört zur inneren Voraussetzung der menschlichen Tat" (Gerhardt 1995, 100).

\section{Das genealogische Geschichtsbewusstsein}

In welcher Weise kann nun die Historie dem Leben als genealogisches Geschichtsbewusstsein dienen?

Zunächst einmal unterscheidet Nietzsche zwischen dem unhistorischen Menschen, der sich nicht erinnern kann und daher keinen Geschichtsbezug hat, dem überhistorischen Menschen, der sich übersättigt und angeekelt von der Geschichte abwendet und dem historischen Menschen, der die Geschichte für sich nutzt. Je nach Zielsetzung und Charakter hat die Geschichte einen ganz unterschiedlich Sinn.

Nietzsche selbst entwickelt drei Perspektiven, die monumentalische, die dem Tätigen und strebenden zugeordnet ist, die antiquarische, die den Bewahrenden und Verehrenden auffängt und die kritische, die dem Leidenden und Bedürftigem Befreiung verspricht.

\subsection{Die monumentalische Betrachtungsweise}

Menschen, die neue Wege gehen und auf Widerstand stoßen oder durch Abwertung entmutigt werden entdecken in der Geschichte „Vorbilder, Lehrer, Tröster“, die sie in ihrem Umfeld und „in der Gegenwart nicht zu finden vermögen“. Diese Orientierung an historische Vorbilder, wenn es keine Gleichgesinnten gibt, greift Nietzsche in der monumentalischen Geschichtsbetrachtung auf:

Die Geschichte gehört vor Allem dem Thätigen und Mächtigem, dem der einen grossen Kampf kämpft ... und die Geschichte als Mittel gegen die Resignation gebraucht (KSA 1, 258).

Der Nachteil dieser monumentalen Betrachtungsweise, die nur die Gipfel in der Landschaft sieht, liegt darin, dass hierbei ebenso der geschichtliche Kontext von Ursachen und Wirkung unbeachtet bleibt, wie die genealogische Verfolgung der Entwicklung von Lebensläufen in ihrer Einbettung in die sozialen und politischen Hintergründe.

Auch die Kinder- und Jugendliteratur, in der Lebensläufe sogenannter großer Menschen auf einfache Weise als Vorbilder präsentiert werden, folgt diesem Prinzip.

Wird die Vergangenheit dabei aber als nachahmenswürdig und nachahmbar beschrieben, steht sie in der Gefahr, „ins Schöne“ umgedeutet zu 287 
werden und einer „mystischen Fiktion“ angenähert zu werden. Regiert also die monumentalische Betrachtung des Vergangenen, über die antiquarische, und die kritische, d.h. bleiben wichtige Faktoren unbeachtet, so leidet die Vergangenheit selbst Schaden:

... große Teile derselben werden vergessen, verachtet, und fließen fort wie eine graue ununterbrochene Flut, und nur einzelne geschmückte Fakten heben sich als Inseln heraus: an den seltenen Personen, die überhaupt sichtbar werden, fällt etwas Unnatürliches und Wunderbares in die Augen, gleichsam die goldene Hüfte, welche die Schüler des Pythagoras an ihrem Meister erkennen wollten. Die monumentale Historie täuscht durch Analogien: sie reizt mit verführerischen Ähnlichkeiten den Mutigen zur Verwegenheit, den Begeisterten zum Fanatismus; und denkt man sich gar diese Historie in den Händen und Köpfen der begabten Egoisten und der schwärmerischen Bösewichter, so werden Reiche zerstört, Fürsten ermordet, Kriege und Revolutionen angestiftet und die Zahl der geschichtlichen „Effekte an sich“, das heißt der Wirkungen ohne zureichende Ursachen, von neuem vermehrt (KSA 1, 262).

Der Wert der monumentalischen Betrachtungsweise ist von der Zielsetzung und den Wertvorstellungen der Probanden abhängig. Diese Geschichtsauffassung regt nicht unbedingt dazu an, das eigene Machtstreben zu hinterfragen oder in einen gesamtgesellschaftlichen Nutzzusammenhang zu stellen, sondern fördert eher die Verfolgung eigner ehrgeiziger Ziele. Die ständig lauernde Gefahr, dass die ursprünglich lebensdienliche Ermutigung zur eigenen Individuation in exzessiven sozialschädlichen Ehrgeiz umkippt, kann durch die Ergänzung mittels der antiquarischen, und kritischen Betrachtungsweise aufgefangen werden.

\subsection{Die antiquarische Betrachtungsweise}

Aus der antiquarische Geschichtsbeschreibung schöpfen vor allem Heimat verbundene Personen Kraft, die sich als Glied einer Kette fühlen, die ihre Wurzeln weit in der Vergangenheit hat und die ihre für sie wertvollen Traditionen bewahren wollen.

Durch diese Pietät trägt er gleichsam den Dank für sein Dasein ab. Indem er das von alters her Bestehende mit behutsamer Hand pflegt, will er die Bedingungen, unter denen er entstanden ist, für solche bewahren, welche nach ihm entstehen sollen-und so dient er dem Leben ... . Die Geschichte seiner Stadt wird ihm zur Geschichte seiner selbst (ibid. 265).

Das Ich dieser Bewahrenden und Ehrenden geht in dem Wir auf und verleiht auch dem einfachen Menschen eine ruhige Würde. Dieser Sinn der Historie gilt 
deshalb vor allem den „minder begünstigten Geschlechtern“, den treuen Sesshaften, die sich in ihrem Umfeld geborgen fühlen, ihr Glück und Wohlgefühl wie Bäume aus ihren Wurzeln, Blüten und Früchten beziehen und nicht abenteuerlich in die Fremde streben. Diese Menschen kommen einer wissensorientierten Geschichtsbetrachtung am nächsten, sie könnten die „Vergangenheit in reines Wissen auflösen“ (ibid.).

Da das Wissen aber nicht wertneutral ist, sondern alles Vergangenen mit einem hohen Wert behaftet ist, verschieben sich die Proportionen, das Neue und Fremde erhält nicht die ihm zustehende Aufmerksamkeit und Achtung, sondern wird angefeindet und abgelehnt. Diese Art der Geschichtsbetrachtung birgt die Gefahr der Erstarrung in sich, sie fördert lediglich das Bewahren und Verstehen nicht aber die schöpferische Weiterentwicklung.

\subsection{Die kritische Betrachtungsweise}

Durch die Gefahren, die aus der monumentalischen und antiquarischen Betrachtung des Vergangenen entstehen können, wird es deutlich, dass der Mensch einer weiteren genealogischen Betrachtungsweise bedarf, die mit ihrem kritischen Blick die Vergangenheit entzaubert. Auch dieser Umgang mit der Historie soll im Dienste des Lebens stehen. Der Mensch

muss die Kraft haben ... eine Vergangenheit zu zerbrechen und aufzulösen, um leben zu können: dies erreicht er dadurch, dass er sie vor Gericht zieht, peinlich inquirirt und endlich verurtheilt; jede Vergangenheit aber ist es werth, verurtheilt zu werden-denn ... immer ist in ihnen die menschliche Gewalt und Schwäche mächtig gewesen. Es ist nicht die Gerechtigkeit, die hier zu Gericht sitz; es ist noch weniger die Gnade, die hier das Urtheil verkündigt: sondern das Leben allein ... aber in den meisten Fällen würde der Spruch ebenso ausfallen, wenn ihn die Gerechtigkeit selbst ausspräche (ibid. 269).

Nach sorgfältiger Überprüfung kann es auf diese Weise klar werden, wie ungerecht die Existenz irgendeins Dinges, Privilegiums, einer Kaste, oder einer Sitte ist und das es sinnvoller ist, sich davon zu trennen, sei es durch Vergessen, Abschaffen oder Vernichten. Obwohl die Notwendigkeit dieses Vorgangs nach der kritischen Prüfung erwiesen ist, sind die Menschen, die ihn vollziehen, nach Nietzsche gefährlich und gefährdet, denn sie durchtrennen mit dem Messer die Wurzeln, aus denen wir hervorgegangen sind.

Eine genealogische Betrachtung sollte deshalb stets alle drei Sinnformen der Geschichte im Auge behalten. $\mathrm{Zu}$ beachten ist auch, dass die monumentalische, die antiquarische und die kritische Geschichtsbeschreibung selbst Momente der historischen Dynamik sind, die in ihrer Gesamtheit entsprechend der jeweiligen Bedürfnisse und Interessen genealogisch erschlossen werden. Beim genealogischen Prinzip werden nicht klare Ursachen-Wirkungen präzise abgeleitet wie im naturwissenschaftlichen Paradigma. Vielmehr geht es bei der genealogischen Methode darum, die 289 
Herkunft der komplexen Entwicklungsmuster zu betrachten und die losen Fäden auf ihre Verwandtschaft hin zu untersuchen.

Michel Foucault greift diese Methode in seinem Werk Überwachen und Strafen. Die Geburt des Gefängnisses (dt. 1994) auf, um den kulturellen Horizont der Gegenwart in Distanz zum Betrachter zu setzen so, dass der Mensch in seiner Gewordenheit durchschaubar und als bloß kontingente Bedingung hinterfragt werden kann (Honneth 2001). Im Rahmen seiner Gesellschaftskritik wehrt er sich wie Nietzsche gegen einen verkürzten Vernunftbegriff. Auch die Feministinnen Judith Butler $(1990,1993)$ und Gesa Lindemann (1993) bedienten sich bei ihrer Geschlechterforschung zur Bedeutungsklärung von Leibsein und Körperhaben Nietzsches genealogischer Methode, die sie mit dem begriffsanalytisch geprägten, mikrosoziologischen Konstruktivismus und Dekonstruktivismus verbanden.

\section{Die zugrunde liegende Wahrheitstheorie}

Eine hohe Bedeutsamkeit erlangt die genealogische Methode bei Nietzsches Wahrheitssuche. Mit ihrer Hilfe entlarvt Nietzsche in seiner Schrift Über Lüge und Wahrheit im außermoralischen Sinn die Begriffe als freie Erfindungen des Menschen:

Als Baugenie hebt sich der Mensch weit über die Biene: diese baut mit Wachs, das sie aus der Natur zusammenholt, er aus dem weit zarteren Stoff der Begriffe, die er erst fabrizieren muß (KSA I, 882).

Der Mensch konstruiert sich die Welt, in der er lebt. Wir glauben zwar etwas von den Dingen zu wissen, können aber letztendlich nur metaphorisch über sie sprechen (ibid. 878). Das „Ding an sich“(ibid.) ist nicht fassbar. Auf die Frage: „Was ist also Wahrheit?“ antwortet Nietzsche:

Ein bewegliches Heer von Metaphern, Metonymien, Anthropomorphismen, kurz eine Summe von menschlichen Relationen .... Die Wahrheiten sind Illusionen, von denen man vergessen hat, dass sie welche sind (ibid. 880).

Die deklarierten Wahrheiten sind deshalb für Nietzsche anthropomorph, sie enthalten „keinen einzigen Punkt, der an sich wahr wäre, wirklich und allgemeingültig" (ibid. 883). Nietzsches Allaussage, dass es keine Wahrheit gibt, lässt sich als Warnung vor einer metaphysischen Objektivierung der Realität interpretieren. Zum Wesen der Dinge kommen wir nicht mit Hilfe der Sprache:

Das ganze Material ... womit später der Mensch der Wahrheit, der Forscher, der Philosoph arbeitet und baut, stammt, wenn nicht aus 
Wolkenkuckucksheim, so doch jedenfalls nicht aus dem Wesen der Dinge (ibid.).

Metaphysisch betrachtet entspricht dem, was wir als "wahr" behaupten, nichts (vgl. Gerhardt 1995, 110). Nietzsche relativiert also die menschliche Erkenntnisfähigkeit durch seine enttäuschende Einsicht, dass wir mit unserer (menschlichen Wahrheit) nicht über uns hinausgelangen. Trotz aller unserer theoretischen Anstrengungen stoßen wir also zu nichts das den Titel eines "Seins" wahrhaft verdient. Volker Gerhardt kommentiert diesen Gedanken mit dem Bild: „Mit dem Finger der Wahrheit zeigen wir letztlich nur auf uns selbst“ (ibid. 106). An die Wahrheit außerhalb seines eigenen Seins, an das „Ewige, Unveränderliche“, kommt der Mensch also nicht heran: „Mit anderen Worten: Im absoluten Sinne gibt es keine Wahrheit" (ibid.).

Das Eingeständnis dieser Erkenntnis führt bei den Wahrheits-Suchenden, die im Wissen darum, dass es für uns Menschen eben keine „erkennbare“ Wahrheit außerhalb unseres eigenen Seins gibt, zu einer Haltung des gegenseitigen Respekts. Niemand kann dann nämlich beanspruchen, die Wahrheit zu besitzen. Das impliziert auch, dass die allzu einseitige Dominanz der Erwachsenen gegenüber den Kindern bei der Antwortsuche auf die "großen" metaphysischen Fragen im strengen Sinne nicht haltbar ist, eine Position, die zu dem grundlegenden Überzeugungscorpus der Bewegung Philosophieren mit Kindern gehört (Werner 1997, 18). Kinder wie Erwachsene zeigen „mit dem Finger der Wahrheit auf sich selbst" und finden daher auch für sich selbst „passende“ Antworten, mit denen sie sich gegenseitig anregen können.

Insgesamt geht es Nietzsche aber weniger um die theoretische Wahrheit, ihn fesselt vielmehr die praktische Bedeutung, die die Wahrheit für den Einzelnen in seinem Lebenszusammenhang hat. Nietzsche fragt: „Wie viel Wahrheit erträgt, wie viel Wahrheit wagt ein Geist?-dies wurde für mich zum eigentlichen Werthmesser" (KSA 9, 16/32; 13, 492). Wert und Unwert der Wahrheit stehen für Nietzsche in Beziehung zu der Wahl der angemessenen Lebensziele.

Durch seine Prämissen ist Nietzsche ein bedeutsamer Philosoph im Horizont des Kinderphilosophierens, denn auch hier wird davon ausgegangen, dass wir nicht an die Wahrheit jenseits unserer eigenen Erkenntnis gelangen können, dass Begriffe gemeinsam in einem vernünftigen Argumentationsprozess erarbeitet werden sollen, und dass sie für den einzelnen eine praktische Lebensbedeutung bei der Wertentscheidung erringen müssen. 


\section{Anwendungsskizze der drei geschichtlichen Betrachtungsweisen auf den Dialog mit Kindern}

Die monumentalische Betrachtungsweise lässt sich mit dem Er-Mythos in Platons Politeia einführen. ER ist ein griechischer Soldat, den die Götter irrtümlicherweise für tot halten und vom Schlachtfeld ins Jenseits tragen. Nachdem der Irrtum aufgeklärt ist, darf Er die Seelen auf ihrer reinigenden Wanderung begleiten, damit er später den Menschen davon berichten kann. Er beobachtet, dass die drei Moiren auf jede Seele ein Lebenslos werfen, das diese dazu befugt, sich aus den verschiedenen Lebensclips einen auszuwählen. So entscheidet sich z.B. Odysseus für das Leben eines einfachen Bauern.

Die Kinder könnten sich nun überlegen, welches Leben sie auswählen würden und sich dabei auf eine ihnen aus den Medien bekannte Biographie beziehen. Der philosophische Dialog könnte die Glücksmomente dieses Lebens thematisieren, den Umgang mit Widerfahrnissen, sowie den Sinn dieses und ihres eigene Lebens.

Die antiquarische Betrachtungsweise könnte über eigene Erlebnisse und Geschichten in ihrer Lebenswelt erschlossen werden. Hierzu könne auch lebendig Zeitzeugen wie Großeltern oder lokale Größen eingeladen werden. Dialogisch kann die Community of Inquiry die Entwicklung von Kultur nachverfolgen und die Stellung, die der einzelnen in seinem Sozialgefüge innehat. Auch die Einflussmöglichkeiten der Politik könnten sich die Kinder hier erschließen.

Die höchste Anforderung an die Kinder stellt die kritische Betrachtungsweise, da sie mit belastenden Inhalten verbunden ist, wie z.B. politische Zeitzeugnisse aus den Kriegen, aus der vordemokratische Vergangenheit, Ereignisse in der Schulgeschichte etc.

Damit aber diese historischen Betrachtungsweisen mit den Kindern vollzogen werden können, müssen sie über ein geschichtliches Bewusstsein verfügen. Sind aber Kinder bereits in der Lage, genealogische Prozesse zu eruieren und zu erfassen? Hier streiten sich die Entwicklungspsychologen.

\section{Können Kinder geschichtlich denken?'}

Die Frage, wie Grundschulkinder mit Geschichte umgehen lernen, über Geschichte denken und historisch lernen können, gehört weitgehend zu den Forschungsdesideraten. Heidrun Dierk (2005) geht dieser Frage nach und kommt zu folgenden Ergebnissen: Dass in den gängigen Bildungsplänen der Geschichtsunterricht erst in der Klassenstufe 7 beginnt ist das missverstandene Resultat einiger älterer Untersuchungen, vor allem der von Roth und Küppers.

${ }^{1}$ Die drei folgenden Kapitel sind Zusammenfassungen von Heidrun Dierk Children Becoming Historians - Innovatives historisches Lernen. 
Heinrich Roth hat in seiner Untersuchung Kind und Geschichte dargelegt, dass das Kind „sich ... der Geschichte das seiner Verstehensstufe Gemäße aneignet" (Roth 1968, 65). Nach Roth vollzieht sich die Entfaltung des geschichtlichen Bewusstseins in Abhängigkeit von der kognitiven Entwicklung dreistufig: Vom Interesse an Fakten über die Erschließung von größeren Sinnzusammenhängen hin zur Frage der existenziellen Betroffenheit, die frühestens mit der Pubertät möglich wird (ibid. 65). Auf Grund seines entwicklungspsychologischen Elementarisierungskonzeptes, hat Roth seit den fünfziger Jahren nachhaltige Wirkung auf die Geschichtsdidaktik. Roth plädierte für eine Individualisierung des Überindividuellen und für eine Reduktion des Komplizierten auf einfache Handlungen (ibid. 110) und zwar mittels der geschichtlichen Erzählung als Verstehenshilfe (ibid. 81, 111).

1961 legte Waltraut Küppers (1966 [1961]) eine empirische Untersuchung Zur Psychologie des Geschichtsunterrichts vor. Sie kommt darin zu dem Ergebnis, dass sich die Hinführung zu vollem geschichtlichem Bewusstsein im Sinne einer verantwortlichen Teilhabe über die Stufen "geschichtliches Interesse - geschichtliches Wissen - geschichtliches Verständnis - geschichtliches Bewusstsein" vollziehe. Ähnlich wie Roth konstatiert sie bei allen Altersstufen ein großes Interesse an der historischen Persönlichkeit (ibid. 91). Auch Küppers unterscheidet drei Stufen des historischen Wissens. Im 5./6. Schuljahr sei nur ein vordergründiges, eindimensionales Erfassen des geschichtlichen Stoffes möglich. Hier habe die emotionale Beteiligung einen großen Stellenwert. Ab dem 7./8. Schuljahr beginne die Fähigkeit zur Tiefengliederung, so dass Wirkungen geschichtlicher Ereignisse erfasst werden können. Auf der 3. Stufe (9./12. Schuljahr) könne Geschichte als vielmaschiges Netz erkannt werden, so dass auch ein Verstehen abstrakter Ordnungen möglich wird (ibid. 122).

Beeinflusst werden diese Ergebnisse durch Küppers' Differenzierung zwischen affinen und diffugen Stoffen des Geschichtsunterrichts in Hinblick auf die Rezeptionsmöglichkeiten der Schülerinnen und Schüler. Während Personen, handlungsstarke Ereignisse und Gegenstände affin sind, da sie mit den frühkindlichen Erfahrungen der Welterschließung korrelieren (ibid. 96, 104, 107) zählen soziale Beziehungen und staatliche Ordnungen zu den diffugen Stoffen, da sie ein Denken auf abstrakter Ebene voraussetzen (ibid. 114) und letztlich nicht vereinfacht werden können (ibid. 117). Aus ihren Forschungsergebnissen zieht Küppers in Bezug auf den Geschichtsunterricht die gleichen Konsequenzen, wie Roth. Deshalb geht sie von der Notwendigkeit des Transponierens aus, d.h. der Überführung des Vergangenen, Rationalen und Abstrakten in Gegenwärtiges, Emotionales und Konkretes (ibid. 127).

Roth und Küppers haben weit reichende Wirkung auf die Geschichtsdidaktik, denn auf der Basis ihrer Ergebnisse, dass nämlich ein 293 
Erfassen geschichtlicher Zusammenhänge erst ab dem 13. Lebensjahr möglich sei, lassen viele Bildungspläne, so z.B. in Baden-Württemberg, den Geschichtsunterricht in der Regel erst ab Klasse 7 beginnen. Dies ist nach meinem Dafürhalten ein Missverständnis der Ergebnisse, denn sowohl Roth als auch Küppers haben den „Vorstufen“ historischen Lernens einen eigenen Wert zugestanden, ohne dies jedoch in ihren Entwürfen didaktisch fruchtbar zu machen.

Inzwischen hat sich die Forschungslage zumindest ansatzweise gewandelt. Inzwischen wird die Meinung vertreten, dass auch Grundschulkinder reflektiert mit Geschichte umgehen. ${ }^{2}$ Diese Sichtweise steht in engem Kontext mit einer Wahrnehmung historischer Kompetenzen von Grundschulkindern, im Sinne eines „bereichsspezifischen Wissens“. Es wird vermutet, dass Kinder mehr oder weniger brauchbare „naive“ subjektive Theorien über Wissensbereiche (domains) haben. Ihre Wissensstrukturen können sie im Sinne der Äqulibrationstheorie von Piaget durch die Konfrontation und Verarbeitung neuer Informationen in der Schule radikal verändern, -ein Vorgang, der als "Conceptual change/Wissensveränderung“ bezeichnet wird.

Im Geschichtsunterricht könnte sich derartiges ereignen, wenn Alltagswissen-aus Erzählungen in der Familie oder gesehenen Fernsehsendungen-durch den Unterricht grundlegend verändert würde und wenn Vorwissen (z.B. zum Nationalsozialismus) aufgenommen und erweitert wird. ... Im Vordergrund stehen deshalb nicht die sogenannten Entwicklungsstufen, sondern die assimilative Annäherung an den Gegenstand (Krieger 2005, 47).

Zwar ist diese instruktionspsychologische Einsicht faktisch nicht neu, denn auf das Vorwissen der Lernenden zurückzugreifen, gehört zu den Grundprinzipien unterrichtlichen Handelns. In Bezug auf den (frühen) Geschichtsunterricht kann man jedoch durchaus von einem Perspektivenwechsel sprechen. Die für den Geschichtsunterricht bedeutendste Konsequenz liegt in der Vorstellung, dass jedes Kind mit jedem Stoff auf jeder Stufe seiner Entwicklung konfrontiert werden kann - sofern die Erschließungswege angemessen sind. Angemessene Erschließungswege sind nach meinem Dafürhalten vor allem aneignungsorientierte Lernformen, die auf der Selbsttätigkeit der Lernenden basieren und die im gemeinsamen Prozess philosophische reflektiert werden.

2 So der Titel eines Aufsatzes von Waltraud Schreiber: "Grundschulkinder gehen reflektiert mit Geschichte um!" (Grundschule 9/2000, 9-11). 


\section{Entwicklung historischer Kompetenzen durch aneignungsorientierte Lernformen}

Als aneignungsorientierte Lernformen lassen sich diejenigen Lernwege verstehen, die eine aktive Teilnahme der Lernenden am Lernprozess fördern und fordern, so, dass diese selbstverantwortliche Subjekte der jeweiligen Lernprozesse werden bzw. sind. Darunter sind alle Methoden des offenen Lernens, handlungsorientierten und kreativen Arbeitens zu subsumieren, die im philosophischen Dialog zur Bildung werden³. In diesem Rahmen ist auch eine kindgemäße Annäherung an die Geschichte möglich. Dabei darf jedoch keineswegs auf die wissenschaftlichen Standards der Disziplin Geschichte verzichtet werden. Das bedeutet nach Ansicht Waltraud Schreibers die Beachtung folgender zwei Kriterien:

Erstens muss der Geschichtsunterricht die „Schülerinnen und Schüler mit solchen Rekonstruktionen von Vergangenheit in Kontakt bringen, die durch die Kontrolle der Disziplin gegangen sind. Weil Schülerinnen und Schüler lebensweltlich mit einer großen Zahl ausgesprochen unterschiedlicher Geschichten in Berührung kommen, muss der Geschichtsunterricht sie befähigen, selbst $\mathrm{zu}$ entscheiden, mit welcher Art von »Geschichte« sie es zu tun haben (Schreiber 2000, 9).

Demnach ist den Schülerinnen und Schülern transparent zu machen, in welcher Weise "Geschichte“ aus der Gegenwart rekonstruierte Vergangenheit ist, bzw. die Schülerinnen und Schüler selbstständig entdecken zu lassen, inwiefern Geschichte Re-Konstruktion aus Quellen und Konstruktion unter gegenwärtigen Fragestellungen ist. Auf diese Weise können die Kinder grundlegende Kompetenzen historischen Lernens entwickeln, ausbilden und anwenden. Mit anderen Worten, man muss die Schülerinnen und Schüler selbst historisch arbeiten lassen. Am Maßgeblichsten für den historischen Lernprozess erscheinen folgende Kompetenzen (ibid. 10).

- die Kompetenz, historisch zu fragen, d.i. echte Fragen nach der Vorgeschichte und dem Fremden in der Vergangenheit zu formulieren. Dies impliziert, dass die Schülerinnen und Schüler mit einem Problem konfrontiert werden, das sie nur unter Rückgriff auf die Vergangenheit lösen können.

- Einher damit geht die Kompetenz, historische Methoden anzuwenden, was vor allem bedeutet, dass die Kinder lernen, auf elementare Weise die Erschließung von Quellen nachzuvollziehen. D.h. es geht hier um die Fähigkeit, Geschichte als „Re-Konstruktion“ zu erkennen.

${ }^{3}$ Dieser Aspekt ist gerade in unserer medial dominierten Zeit, die an den Erlebnissen an der primären Welt immer ärmer wird, besonders betonenswert, vgl. Weschenfelder (1992) und Günzler (2002). 
- Eine Art Komplementärbewegung dazu ist die Kompetenz, fertige Geschichten (z.B. Historienfilme, Historienromane, Darstellungen in historischen Sachbüchern) als Konstruktion zu erkennen, d.h. sie zu dekonstruieren, indem die fertigen Geschichten in Hinblick auf ihre Triftigkeit überprüft werden.

- Die Kompetenz, das Erarbeitete auf die eigene Weltsicht zu übertragen, bezieht sich auf die grundlegende Orientierungsfunktion, die eine Beschäftigung mit der Geschichte erreichen will. Ob sich aus dem erworbenen Geschichtsbewusstsein Verhaltensänderungen der Lernenden in ihrem Alltag ergeben, ist schwer zu überprüfen und bleibt wohl eher ein Ideal des Geschichtsunterrichts, getreu der schon in der Antike propagierten Zielsetzung der „Historia magistra vitae“, der auch der Graecophile Nietzsche anhängt.

Wenn es durch den Umgang mit Geschichte in der Schule gelingt, dass bei Grundschülerinnen und -schülern diese Kompetenzen angebahnt und gefördert werden, dann kann ein reflektierter Umgang mit der Geschichte möglich werden. Zugleich wird in elementarer Weise ein Einblick in geschichtsphilosophische Grundlagen gewährt, nämlich Geschichte als „story“ (Danto) zu verstehen. Durch historisches Arbeiten können Kinder lernen, die Wirklichkeit vertieft zu durchschauen.

In welcher Weise dies praktisch umgesetzt werden kann, soll nun drei Beispielen aus der Literatur gezeigt werden.

\section{Beispiel 1: Zeitzeugenbefragung im Rahmen eines Projekts zur Schulgeschichte}

Zeitzeugen können Personen, Quellen, oder historische Gegenstände sein. In einer Untersuchung aus den 80er Jahren hat Alistair Ross gezeigt, dass Kinder der Primarstufe im Rahmen eines Projekts zur Schulgeschichte historische Kompetenzen entwickeln können (Ross 1998, 432-447). Kinder der Klassenstufen 2 und 4 befragten Zeitzeuginnen und -zeugen zur Evakuierung Londoner Schulkinder zu Beginn des 2. WK in den Ort Lacock. Die Befragungsergebnisse dokumentierten sie nach Maßgabe ihrer Fähigkeiten.

Durch die Befragung und die z.T. sehr widersprüchlichen Aussagen wurden die Kinder an quellenkritische Überlegungen herangeführt. Sie erkannten, dass Menschen Sachverhalte in der Retrospektive unterschiedlich darstellen und beurteilen, dass sie unterschiedliche Ereignisse für erwähnenswert halten, dass durch den zeitlichen Abstand Erinnerungen verändert werden usw.

Hier ein Auszug aus dem Gesprächsprotokoll (ibid. 442):

Teacher: Warum sind die Geschichten verschieden? 
Tom: $\quad$ Es könnten verschiedene Plätze gewesen sein in unterschiedlichen Teilen von Lacock.

Martha; $\quad$ Aber Lacock ist überhaupt nicht groß!

Wanda: Das ist nicht möglich.

Tania: $\quad$ Fr. B. sagte, dass sie nicht direkt in der Stadt lebte, sie lebte ungefähr eine Meile entfernt.

Sam: $\quad$ Sie könnten sich einfach an verschiedene Dinge erinnert haben. Vielleicht gab es viel Freundlichkeit, viele Kämpfe und Menschen erinnerten sich unterschiedlich.

Tom: $\quad$ Hr. E. erzählte uns, dass sie ein Konzert hatten, und nach dem Konzert waren aufgrund von diesem alle freundlich. Vielleicht erinnerte sich Hr. H. an die Zeit nach dem Konzert und die anderen an die Zeit davor.

$\cdots$

Dann besprach die Klasse, wie sie herausfinden konnte, warum sich die Einzelnen an verschiedene Versionen erinnerten.

Die Lehrerin fragte nach alternativen greifbaren Quellen, mit deren Hilfe sie dei Ereignisse kontrollieren konnten:

Die Lehrerin fragte, wie das Logbuch als Quelle mit den Erinnerungen der Menschen, die sie interviewt hatten, vergleichbar sein ich, würde?

Shagufta: $\quad$ Nun ja, reden, es mag sein, dass sie sich nicht erinnern.

Teacher: $\quad$ An welche Art von Dinge denkt ihr, erinnern sich Menschen am besten?

Sam: $\quad$ Dinge, die etwas mit ihnen zu tun haben, mehr als an Dingen die draußen passieren.

May-ai: Ja.

Berta: $\quad$ Wie der Schweine? Pig smash (der Stank)

Sam: [Im Logbuch] Aber sie mussten sich nicht zurück erinnern. Sie schrieben es eigentlich nieder, während sie dort waren.

Emily: $\quad$ Es steckt mehr Beweis im Logbuch, sie schrieben es zu dieser Zeit.

Teacher: Ist das was im Logbuch geschrieben steht zwangsläufig alles was passiert ist?

Child: $\quad$ Nein.

Sam: $\quad$ Weil wenn du alles niederschreiben würdest, würden es hunderte von Seiten sein.

Emily: $\quad$ Es ist die Schulakte, nicht von außerhalb der Schule.

Edmont: $\quad$ Es ist der Schulleiter. Was er weiß.

Teacher: $\quad$ Gibt es irgendeinen Grund warum der Schulleiter über 
etwas Bescheid wissen sollte und es nicht niederschreiben würde?

Sam: Wenn es wirklich schlimm wäre, dann würden vielleicht die Leute, die es lesen denken es war eine böse/ schlechte Schule.

Andre: $\quad$ Wenn er niederschreiben würde, dass es nichts anderes gab außer Kämpfe.

Berta: $\quad$ Er würde nicht alles erzählen, aber er würde das meiste davon erzählen.

Emily: $\quad$ Er mag Dinge erzählen, aber er könnte diese eine wenig drehen, er könnte sozusagen die Wahrheit drehen, wenn es also wirklich schlimm wäre, ein schlimmer Kampf und jemand wurde richtig verletzt, würde er vielleicht sagen, dass es einen Kampf gab, aber er würde es ein bisschen umändern und sagen "ein aufgeschürftes Knie" oder etwas in der Art.

Deutlich wird erkennbar, dass die Kinder quellenkritisch denken können und dass die Selektivität die Erinnerung am konkreten Beispiel sichtbar wird. Die Kinder vermuten hierfür unterschiedliche Ursachen: Lange zurück liegende Ereignisse können möglicherweise nicht mehr exakt erinnert werden, Erinnerung können je nach Adressatenbezug verändert, z.B. geschönt, werden. Auch ist den Kinder $\mathrm{n}$ bewusst, dass die Erinnerung eines Menschen an vergangene Ereignisse eine andere Validität hat als eine zeitgleich schriftlich fixierte Quelle.

Dieser Prozess wurde den Kinder noch einmal bei Ihrer eigenen Quellenniederschrift deutlich: Auch sie selbst trafen die Auswahl dessen, was sie niederschrieben, und zwar hinsichtlich unterschiedlicher Kriterien, z.B. nach der Häufigkeit des Berichteten, dem vermuteten Interesse der Lesenden oder unter regionalspezifischer Perspektive, d.h. sie notierten das nicht Generalisierbare (ibid. 444). Ross kommt $\mathrm{zu}$ folgendem, ermutigenden Ergebnis seines Projekts:

These younger children had also developed a critical awareness of the nature of evidence, and were prepared to justify different kinds of sources as having varying degrees of reliability. However, they seemed less able to accommodate the idea of bias. ... The older children seemed much more prepared to accept that people's accounts could be biased, and that this would need to be taken into account when conflicting evidence was found. They were also aware that they themselves were engaged in a progress of selecting evidence. ... By allowing the children to take on a genuine investigation in this way, giving them access to real data, we 
encouraged them to act as historians. It seems that the best way to acquire the skills and attitudes that historians have is to practice them in a real enquiry (Ross, ibid. 446).

Exemplarisch belegt das von Ross geschilderte Beispiel, dass schon Grundschulkinder im Rahmen ihrer Verstehensmöglichkeiten historisch lernen und arbeiten können. Ein solches Arbeiten ist am besten als „Lernen der community of inquiry im Geschichtsraum“ zu beschreiben, als historisches Lernen im offenen Raum innerhalb und außerhalb der Schule, in dem im gemeinsam Dialog Methoden, Kommunikation und Innovation eingeübt werden (Rohrbach 2005).

\section{Beispiel 2: Historischer Erkenntnisgewinn im Diskurs}

Ein anderes Beispiel dafür, dass schon Grundschulkinder zu historischen Erkenntnissen auf genuin historischen Wegen fähig sind, zeigen Untersuchungen zur Diskussionsfähigkeit von Grundschulkindern. Martina Länger-Plan (2002, 31-33) beschreibt eine Untersuchung von 9-10jährigen italienischen Kindern:

Die Auswertung der transkribierten Dialoge zeigt, dass auch im Grundschulalter ein hohes Diskussionsniveau erreicht werden kann. So besprachen die Kinder etwa die Frage, inwiefern die Hunnen als "nicht normal" gelten können, ob sie nur anders waren oder als minderwertig angesehen werden müssten. Dabei versuchten sie multiperspektivisch vorzugehen und Gründe dafür zu finden, weshalb die Hunnen bestimmte Aktionen vornehmen. Außerdem stellten sie Überlegungen über die Situation des Historikers an, z.B. ob man zur Zeit eines Ereignisses gelebt haben muss, um eine Geschichtsdarstellung darüber schreiben zu können, und wie es mit seiner Verpflichtung ausgesehen hätte, die Wahrheit zu schreiben. Zudem machten sie sich Gedanken über die Entstehung des Dokumentes. Interessant war vor allem, dass-auch wenn die Diskussionen bisweilen stockten und Hilfestellungen nötig gewesen wären-insgesamt ein höheres Niveau erreicht wurde als bei anderen Untersuchungen, in denen Lehrer die Diskussionen leiteten.

Freilich lässt sich dies nur durchführen, wenn zugelassen wird, dass die Kinder tatsächlich auch von ihrem Alltagsverständnis aus diskutieren dürfen, um sich so dem historischen Fremdverstehen annähern zu können. Selbst wenn die Ergebnissen im Detail bisweilen nicht angemessen waren, so zeigt doch die Bandbreite der aufgeworfenen Fragen, dass die historische Problematik im Kern richtig erfasst wird.

Das hier vorgelegte Beispiel macht deutlich, dass Grundschulkinder durchaus ein historisches Interesse haben und ein historisches Bewusstsein entwickeln können, dass die Frage nach dem, was faktisch passiert ist, weit hinter sich lässt. Vielmehr sind sie-vergleichbar dem Oral history-Projekt- 
in der Lage, den Aussagewert einer (hier schriftlichen) Quelle kritisch zu hinterfragen. Dass sie dabei auch auf ihr Alltagswissen über die Tendenz von Darstellungen und Erzählungen zurückgreifen, erscheint evident. Sie erkennen dabei auch, dass historische Darstellungen Interpretationen von Ereignissen sind, die stets vorläufige Interpretationen bleiben.

\section{Beispiel 3. Besseres Verstehen der Gegenwart durch die Vergangenheit}

Als drittes Beispiel soll der japanische Unterricht von Toshiaki Ôse: „Inochi: Was ist das Band der Familie" vorgestellt werden, den er am 19. September 2003 in der vierten Klasse der die Städtische Grundschule Hamanogô in Chigasaki durchgeführt hatte.

Da es immer mehr Kinder gibt, die aufgrund der häuslichen Situation, wie dem Tod eines Elternteils oder der Scheidung der Eltern nach deren Wiederverheiratung in einer neu zusammengesetzten Familie leben, sollte die Unterrichtpraxis zur Reflexion darüber anregen, ob Kinder in neu zusammen gesetzten Familien wieder glücklich werden können. Inspiriert wurde dieser Unterricht (vgl. Dobashi 2007) durch die Ausgrabungsfotos vom Vulkanausbruch des Berges Asama im achtzehnten Jahrhundert, die im dortigen Heimatmuseum ausgestellt sind. Das Museumsmaterial zum Untergang und Wiederaufbau des Dorfes Kambara fasste der Lehrer in einer Impulsgeschichte zusammen:

Am 8. Juli 1783 wurde das Dorf Kambara durch einen Vulkanausbruch des Berges Asama fast vollständig zerstört. Alle anwesenden Bewohner, die ins offene Land flohen, wurden von dem schlammigen Strom aus vulkanischer Asche, Steinen und Felsen überwältigt. Die Kranken oder schwachen Alten, die nicht weit laufen konnten, versuchten zum kleinen nahen Hügel zu fliehen, auf dem der kleine Tempel Kannon-Dô stand. Eine lange Treppe führt zu ihm hinauf. Jeder der es schaffte, bis zur 15. Treppenstufe zu kommen, war gerettet. Die drei und neunzig Überlebenden wurden von den drei Dorfvorstehern auf zwölf neu zusammengesetzte Familien und Häuser aufgeteilt.

Nachdem die Schülerinnen und Schüler mit Papierpuppen und Pappteller, die die zwölf Häuser darstellten, diese Aufteilung rekonstruiert hatten, wurde es still in der Klasse. Plötzlich ertönte von hinten die Stimme Ôses: „Sind die zwölf Familien wohl glücklich gewesen?“ (I, 135). 
Ich zitiere nun einen Protokollauszug des sich daraus entwickelnden Gesprächs:

Kiichi: Waren sie glücklich? Das ist kaum zu glauben

Ôse: Was meinen die anderen?

Makoto: Ich denke, sie waren nicht glücklich. Sie wohnten doch noch nicht lange zusammen, gerade eben hatte etwa die Frau ihren Mann verloren.

Keiko: Sie ist traurig.

Makoto: Sie ist einsam, da sie ihren Mann verloren hat.

Megumi: Die vorherige Familie war besser als die gegenwärtige Familie. Es waren Blutsverwandte. In der gegenwärtigen Familie gibt es keine Blutsverbindung.

Kôta: Ich denke, glücklich sind sie nur darüber, dass sie weiterlebten, aber ansonsten war es eher armselig, deshalb wünschten sie vielleicht aufgrund der Lebensungünstigkeiten neue Umverteilungen

Morita: Bitte, sage das noch einmal.

Kôta: Die insgesamt drei und neunzig Personen bekamen von drei Gemeindevorstehern Häuser geschenkt, die in der Nähe aufgebaut worden waren. Innerhalb der Häuser entstanden dann aber Streitigkeiten, die Einteilung war nicht erfolgreich, aus diesem Grunde wünschte man sich neue Aufteilungen mit anderen Personen. Aber solche Forderung wäre ein Luxus gewesen, man konnte sie nicht aussprechen.

Ôse: Wie denkst Du, wenn es Dich beträfe? Wenn Du damals mit jenen Personen leben müsstest? Wärst Du glücklich?

Kazuya: Vielleicht wäre ich eines Tages glücklich, es gäbe aber sicher auch Tage, die nicht glücklich sind.

Ôse: Warum?

Kazuya: Zwar ist es widerwärtig, aber wenn man nicht in diesem Haus leben würde, müsste man sterben, sowie die Situation war. Deshalb ist es besser, dass man dort lebt, obwohl es dort so ist.

Ôse: Man konnte alleine nicht überleben.

Kazuya: Deshalb müsste man gegenüber den unbekannten Personen gehorsam sein, wenn man das Kind wäre, das dort wohnt.

(KI, 179-181, vgl. I., 135 f.) (Übersetzt von Kiichi Shimoyamada)

Die Kinder waren sehr betroffen. Die meisten Kinder dachten, dass die zwangsweise zusammengesetzten Familien kein echtes Glück empfinden konnten, einige gingen davon aus, dass sie in dieser Situation Selbstmord gemacht hätten, um zur alten Familie zurückzukehren. Trotzdem wurden dann doch zwei Argumente gefunden, warum man trotz allem in der neu 
organisierten Familie Glück empfinden konnte: (1) weil man alleine nicht überleben kann, (2) weil man die Hoffnung haben kann, später wieder eine eigene Familie zu gründen. Ihr Lehrer Ôse erzählte ihnen, dass noch heute in diesem Dorf Enkel leben würden, die aus diesen Familien abstammten. Im anschließenden Gespräch wurden diese historischen Erfahrungen mit den heutigen verglichen.

\section{Abschließende Überlegungen}

Die referierten Beispiele sollen Mut machen, Kinder mit Geschichte zu konfrontieren und die Kinder $\mathrm{zu}$ motivieren, nach historischen Erkenntnisprinzipien $\mathrm{zu}$ fragen. Auf diese Weise wird eine kritische Grundhaltung eingeübt, die sich nicht mit dem Vordergründigen einer Darstellung, zufrieden gibt, sondern nach der Basis der Erkenntnis und den der Darstellung zu Grunde liegenden Interessen fragt.

Die historische Betrachtungsweise impliziert ein vertieftes Verstehen einer Epoche gegenüber ihren Augenzeuginnen und -zeugen sowie Zeitzeugnissen, denn sie vermag einen Teil der Zukunft der Epoche zu berücksichtigen. Damit erhalten Kinder und Jugendliche eine Chance gegenüber den in der Vergangenheit handelnden Menschen, da sie zumindest einen Teil der Handlungsfolgen überblicken und beurteilen werden können. Damit erkennen sie zugleich die zeitgebundene Perspektivität der historischen Betrachtung. ${ }^{4}$ Wenn sie diese auch als eine um ein Stück Zukunft erweiterte Perspektive zu würdigen wissen, so bleibt doch stets das Bewusstsein, von ihrer eigenen Zukunft eingeholt zu werden. Kinder und Jugendliche entwickeln die Erkenntnis, dass jede historische Betrachtung, auch die ihres eigenen Lebens, unabgeschlossen und revisionsbedürftig bleibt.

Dass Schülerinnen und Schüler sich solchen Einsichten nähern können, haben die vorliegenden Beispiele gezeigt. Die Vorstellung, dass Kinder entwicklungsbedingt lediglich an Fakten und Personen interessiert sind, kann somit als widerlegt gelten. Unter entsprechender Anleitung und Motivation können sie tatsächlich historisches Bewusstsein entwickeln und geschichtsphilosophische Fragestellungen formulieren. Diese Fähigkeiten dienen nicht nur historischen Lernprozessen, sondern darüber hinaus einer differenzierten Wahrnehmung der Wirklichkeit und als integrierte Bildung ihrem eigenen Leben.

${ }^{4}$ So ändern sich historische Darstellungen und Beurteilungen nicht lediglich durch eine Veränderung der Quellenlage, sondern auch durch die Entdeckung bisher unerkannt gebliebener Wirkungen von Personen, Ereignissen, usw. 


\section{Literatur}

Bäuml-Roßnagl, M.-A. 2010. "Mit Hugo Kükelhaus auf der BUGA 2005 in München. Sinnesphilosophische Erfahrungen zum Verhältnis von Reflexion und Sinnlichkeit". http://www.hugokuekelhaus.de/cms/images/dokumente/buga2005.pdf

Butler, J. 1990. Gender Trouble. New York: Routledge, Chapman, \& Hall Inc.

Butler, J. 1993. Bodies that Matter: On the Discoursive Limits of Sex. New York: Routledge.

Dierk, H. 2004. "Children Becoming Historians - Innovatives historisches Lernen". In $K P B, 62$.

Dierk, H. 2005. Kirchengeschichte elementar. Münster: LIT Verlag.

Dobashi, T. 2007. „»Inochi« oder die »Endlichkeit des Lebens«. Toshiaki Ôse philosophiert mit japanischen Kindern. In Marsal, E., Dobashi, T., Weber, B., \& Lund, F. G. (Hrsg.), Ethische Reflexionskompetenz im Grundschulalter. Konzepte des Philosophierens mit Kindern. Frankfurt am Main: Peter Lang:355-368.

Dobashi, T. 2007. "Unterricht als Urwissenschaft - Takeji Hayashi und das Philosophieren mit Kindern". In Camhy, D.G. (Hrsg.), Philosophische Grundlagen innovativen Lernens. Sankt Augustin: Academia: 203-210.

Foucault, M. 1994dt. [1975]. Überwachen und Strafen. Die Geburt des Gefängnisses. Frankfurt am Main: Suhrkamp.

Gerhardt, V. 1995. Friedrich Nietzsche. München: Beck.

Günzler, C. 2002. Bildung und Erziehung im Denken Gottes. Köln Wien: Böhlau.

Hayashi, T. \& Itô, K. 1990. Jugyô wo Tsuikyûsuru to Iukoto. Tôkyô: Kokudosha.

Honneth, A. 2001. "Einleitung: Genealogie als Kritik". In Honneth, A. \& Saar, M. (Hrsg.), M. Foucault. Zwischenbilanz einer Rezeption. Frankfurter Foucault-Konferenz 2001. Frankfurt am Main: Suhrkamp.

Itô, K. 1992. Tamashii ni Uttaeru Jugyô. Tôkyô: NHK Books.

Krieger, R. 2005. "Mehr Möglichkeiten als Grenzen - Anmerkungen eines Psychologen". In K. Bergmann \& R. Rohrbach (Hrsg.), Kinder entdecken Geschichte. Theorie und Praxis historischen Lernens in der Grundschule und im frühen Geschichtsunterricht. In Zeitschrift für Geschichtsdidaktik 2: 263-266.

Küppers, W. 1966 [1961]. Zur Psychologie des Geschichtsunterrichts. Eine Untersuchung über Geschichtswissen und Geschichtsverständnis bei Schülern. Stuttgart - Bern: CH Beck.

Lindeman, G. 2011. Das paradoxe Geschlecht. Transsexualität im Spannungsfeld von Körper, Leib und Gefühl. Frankfurt am Main: Springer.

Marsal, E. (Hrsg.) 2006. Karlsruher Pädagogische Beiträge 62. Karlsruhe: PH, März 2006: 105-127. 
Martens, E. 2003. Methodik des Ethik- und Philosophieunterrichts. Philosophieren als elementare Kulturtechnik. Hannover: Siebert.

Mayring, P. 2007. Qualitative Inhaltsanalyse. Grundlagen und Techniken. Weinheim: Beltz UTB.

Milgram, S. 1998. Das Milgram Experiment. Zur Gehorsamsbereitschaft gegenüber Autorität. Reinbek: Rowohlt.

Nietzsche, F. 1967-. Kritische Studienausgabe (KSA), Hrsg. von G. Colli \& M. Montinari. Werke in 15 Bänden. Berlin - New York: Walter de Gruyter.

Ôse, T. 2004. Kagayake Inochi no Jugyô. Tôkyô: Shôgakukan.

Satô, M. 2000. Gakkô wo Tsukuru. Tôkyô: Shôgakuku.

Ross, A. 1998. Equalities and Education in Europe: Explanations and Excuses for Inequaliy. Cambridge: Cambridge Scholar Publishing.

Roth, H. 19685. Kind und Geschichte. Psychologische Voraussetzungen des Geschichtsunterrichts in der Volksschule. Ergänzte Aufl. München: Kösel.

Satô, M. 1996. Karikyuramu no Hihyô. Yokohama: Seshiki Shobô.

Schreiber, W. 2000. "Grundschulkinder gehen reflektiert mit Geschichte um!" Grundschule 9: 9-11.

Tebartz van Elst, A. 1994. Ästhetik der Metapher. Zum Streit zwischen Philosophie und Rhetorik bei Friedrich Nietzsche. Freiburg-München: Verlag Karl Alber.

Weschenfelder, K. 1992. Handbuch Museumspädagogik. Orientierungen und Methoden für die Praxis. Düsseldorf: Schwann. 


\author{
Eva Marsal (Karlsruhe) \\ Takara Dobashi (Hiroshima)
}

\title{
Geschichtsbewusstsein und Zeitzeugnis. Nietzsches Genealogie als Anregung zum philosophischen Dialog mit Kindern \\ Historical Awareness And the Witness of Time. The Nietzschean Genealogy as a Facilitator of Philosophizing with Children
}

\begin{abstract}
The paper confronts the reader with Nietzchean critical approach to history, truth, life, and education. Far away from progressive-euphoric ideologies of $19^{\text {th }}$, the authors consider the following questions: How children live historical occurences when being tought in classrooms (for example in Italy, Japan, etc.), how can we strengthen their ability for reflected relations to history as well as for interconnecting between past, present, and future life? Psychological, pedagogical, and philosophical considerations meet intercultural contexts.
\end{abstract}

Keywords: Nietzsche, teaching history, truth, education, life

Doi:10.14746/eip.2014.2.19 